\title{
Concrete based thermal energy storage for steam generation: A numerical investigation
}

\author{
Shobhana Singh Kim Sørensen \\ Department of Energy Technology, Aalborg University, Pontoppidanstræde 111, 9022, Aalborg, Denmark, \\ \{ssi,kso\} det.aau.dk
}

\begin{abstract}
Establishing enhancement methods to develop cost-effective thermal energy storage technology requires a detailed analysis. In this paper, a numerical investigation of the concrete based thermal energy storage system is carried out. The storage system consists of a heat transfer fluid flowing inside the heat exchanger tubes embedded in a cylindrical shape concrete configuration. Three-dimensional multiphysics model of the storage system is developed to investigate transient conjugate heat transfer between the two mediums, the heat transfer fluid, and the concrete. The model comprehends the dynamics of storing thermal energy in the storage at the temperature range of $350-390{ }^{\circ} \mathrm{C}$. To evaluate the performance of the storage system using finned heat exchanger tubes, thermal characteristics such as charging/discharging time, energy, and exergy efficiency are predicted. The developed model satisfactorily demonstrates the dynamic behavior of the concrete based thermal storage system and its sub-processes, and thus offers a suitable framework for future investigations leading to an easier and more economical solution.
\end{abstract}

Keywords: Dynamic model, thermal energy storage system, heat transfer enhancement, energy and exergy efficiency

\section{Introduction}

Thermal energy storage (TES) technologies have been of great merit in eliminating inconsistencies in the demand and supply of energy. TES stores thermal energy typically from renewable sources, waste heat or surplus energy production by heating or cooling a storage medium for later use in industrial processes mainly for power generation, heating, and cooling in buildings, etc. Moreover, TES minimizes fossil fuel consumption, reduce $\mathrm{CO}_{2}$ emissions and lower the need for costly peak power and heat production capacity. TES systems are extensively categorized into three kinds, namely: sensible heat storage, latent heat storage, and thermo-chemical storage. Concrete thermal energy storage (CTES) is one of the preferable sensible heat storage systems due to low exergy loss, low cost and easy management of the material.
Many research and development projects to demonstrate high temperature TES for concentrated solar power (CSP) have been reported. Solid media thermal storage systems, each with a storage capacity of $350 \mathrm{kWh}$ and a maximum temperature of $390{ }^{\circ} \mathrm{C}$, have been developed by the German Aerospace Center (DLR) for parabolic trough power plants (Tamme et al., 2004; Laing et al., 2006, 2009; Feldhoff et al., 2012). Researchers have also studied the application of TES systems for a direct steam generation (DSG) (Laing et al., 2011; Seitz et al., 2014). A detailed review of case studies on high-temperature thermal energy storage for power generation is reported by Gil et al. (2010) and Medrano et al. (2010). From the reported research, it has become clear that using the sensible heat of solid material to store thermal energy is a promising technology for concentrated solar power plants, however, for DSG, feasible storage designs and heat transfer enhancement techniques are needed to be studied.

Performance improvements are associated mainly with thermo-physical properties of the storage material and design parameters of tubular heat exchanger integrated within that storage material. For a cost-effective TES, it is necessary to select a viable mechanical design with a higher thermodynamic efficiency of the processes involved. Augmentation in heat transfer is one of the enhancement techniques that are used to improve the performance of energy storage systems. Heat transfer augmentation can be achieved by increasing the thermal conductivity of the storage material, by using finned tubes and by dispersing highly conductive particles in the heat transfer fluid (HTF), etc. An experimental study to compare the heat transfer enhancement in a storage system with phase change material (PCM) using circularly and longitudinal finned concentric heat exchanger is reported by Agyenim et al. (2009). They analyzed the heat transfer characteristics using isotherm plots and temperature curves and found that longitudinal finned system performs the best during charging with an insignificant sub-cooling during discharging.

Depending on the application type, CTES requires proper designing to charge and discharge thermal energy which is difficult to anticipate without any prior knowledge of its physical behavior under certain operating conditions. Numerical Heat transfer and fluid 


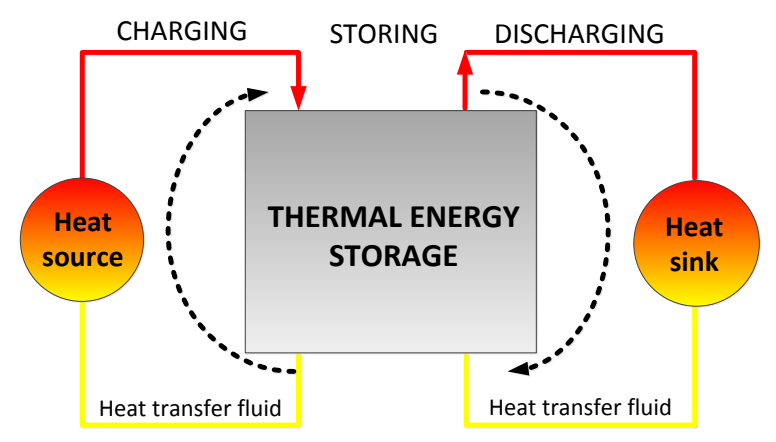

Figure 1. Thermal energy storage system and its sub-process

flow models, in most of the cases, help to understand the critical factors that influence the performance such as variations in spatial and temporal temperature and energy flow, thereby, predict improvements in the system design. Despite this advantage, limited work mainly focused on charging process is available in the literature. Therefore, the aim of this study is to analyze both charging and discharging by developing a three-dimensional (3D) numerical model of CTES to implement a heat transfer enhancement technique for a cost-effective operation. The model is utilized to analyze the influence of finned tubes on storage characteristics during the charging and discharging processes. Determination of the objective variables can help develop a useful framework for future multi-objective optimization of the storage system.

\section{Concrete thermal energy storage system: Design description}

CTES is a sensible heat storage system which stores the thermal energy in concrete as a storage medium. The HTF flows through the tubes and transfers the thermal energy to concrete where it is stored as sensible heat. Figure 1 shows a schematic of a simple storage process where three main sub-processes namely charging, storing and discharging can be seen. The amount of the energy stored in the storage is equal to the temperature rise and the specific heat capacity of the concrete and is governed by Eq.1 as:

$$
Q_{s}=m \cdot C_{p} \cdot \Delta T=\rho \cdot V \cdot C_{p} \cdot \Delta T
$$

where $Q_{s}$ is the amount of thermal energy stored, $m$ is the mass of the storage media, $\rho$ is the density of the storage material, $C_{p}$ is the specific heat of the storage material, $V$ is the volume of storage media and $\Delta T$ is the temperature difference.

The CTES system design investigated in this paper typically consists of a cylindrical concrete unit with a fixed number of embedded heat exchanger tubes. Thermal oil is used as the HTF and the properties are adopted from (Tamme et al., 2004). Figure 2 represents the computational geometry of the CTES unit simulated in the present study. The design can be employed for
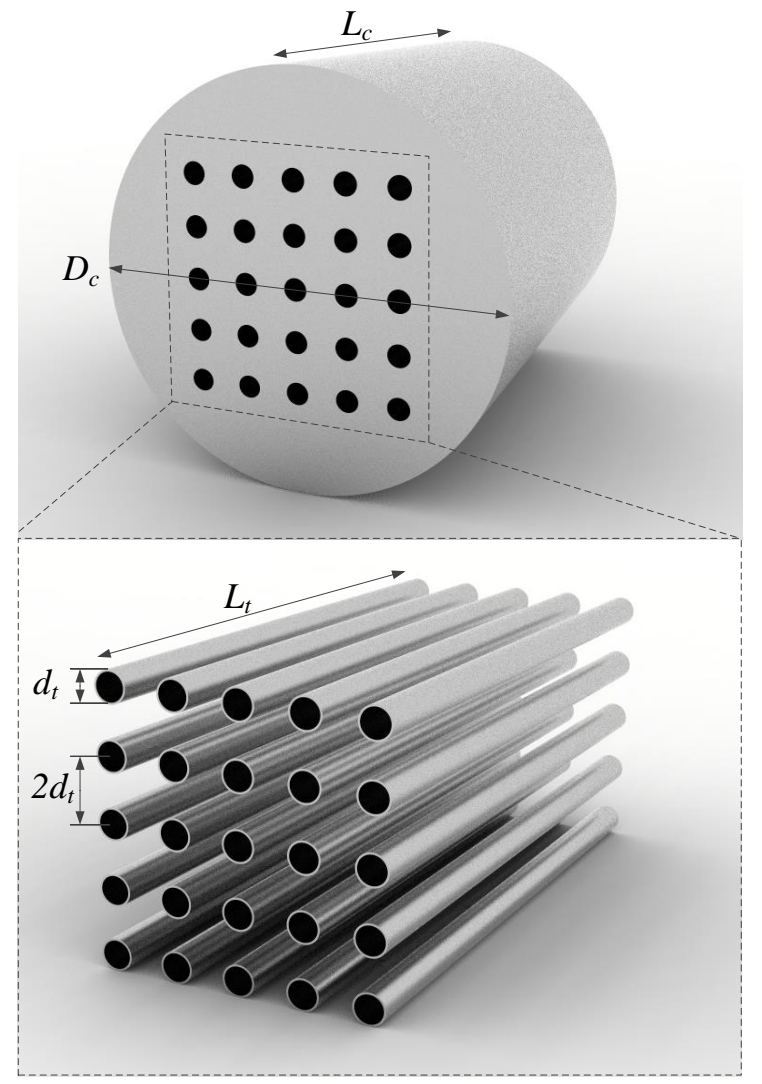

Figure 2. Computational model of the CTES system unit

higher storage capacities when the unit is connected in series to compose a complete module. Longitudinal fins have found to perform better than circular fins in thermal response during charging and discharging (Agyenim et al., 2009); therefore, longitudinal fins are chosen to investigate the impact of finned tubes as shown in Fig.3. The tubes with four longitudinal fins are arranged parallel to the flow direction of HTF. Since the technical feasibility depends upon the system design and excellent thermo-mechanical material properties, high temperature concrete with higher thermal conductivity is used. Table 1 shows the list of system design parameters and thermo-physical properties of the materials used to simulate the system are described in Table 2. The physical processes such as conjugate heat transfer (heat transfer by conduction and convection) and fluid flow in the system are simulated with following assumptions:

- The inlet velocity profile of HTF is fully developed.

- The pressure loss through the tubes is neglected.

- Losses from the system are negligible.

- Concrete properties are isotropic.

- Non-adiabatic storage boundaries. 


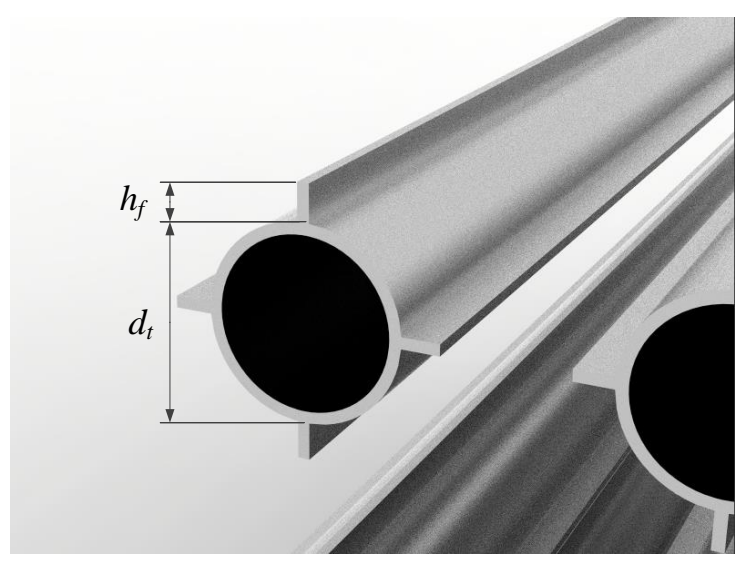

Figure 3. Longitudinal finned tube

\section{Model development}

\subsection{Governing equation and boundary conditions}

The dynamic behavior of the HTF flowing inside the heat exchanger tubes is simulated using the continuity equation and the Navier-Stokes equations as:

$$
\frac{\partial \rho_{f}}{\partial t}+\nabla \cdot\left(\rho_{f} \mathbf{v}\right)=0
$$

where $\rho_{f}$ is the density of the HTF and $\mathbf{v}$ is the velocity vector.

$$
\rho_{f} \frac{\partial \rho_{f}}{\partial t}=-\nabla P+\mu \nabla^{2} \mathbf{v}
$$

where $P$ is the pressure and $\mu$ is the dynamic viscosity of the HTF.

During operation of CTES, convective heat transfer takes place from the flowing HTF to the wall of the tube which is governed by the energy equation as:

$$
\rho_{f} C_{p, f} \frac{\partial T}{\partial t}+\rho_{f} C_{p, f} \mathbf{v} \cdot \nabla T=\nabla \cdot\left(k_{f} \nabla T\right)+Q
$$

where $C_{p, f}$ is the specific heat of HTF, $k_{f}$ is the thermal conductivity of HTF, $T$ is the temperature field in fluid and $Q$ is a source or sink term.

Governing equation for the Simultaneous conductive heat transfer from the heat exchanger tubes to the

Table 1. Design parameters of the CTES system unit

\begin{tabular}{ll}
\hline Property & Value \\
\hline Length of the concrete unit, $L_{\mathrm{t}}$ & $0.5 \mathrm{~m}$ \\
Diameter of the concrete unit, $D_{\mathrm{t}}$ & $0.3 \mathrm{~m}$ \\
Diameter of the tube, $d_{\mathrm{t}}$ & $0.02 \mathrm{~m}$ \\
Length of the tube, $L_{\mathrm{t}}$ & $0.5 \mathrm{~m}$ \\
Height of the fin, $h_{\mathrm{f}}$ & $0.01 \mathrm{~m}$ \\
Thickness of the fin, $\delta_{\mathrm{f}}$ & $0.001 \mathrm{~m}$ \\
\hline
\end{tabular}

Table 2. Thermo-physical properties of materials

\begin{tabular}{llll}
\hline Property & Concrete & $\begin{array}{l}\text { Heat } \\
\text { transfer } \\
\text { fluid }\end{array}$ & $\begin{array}{l}\text { Heat } \\
\text { exchanger } \\
\text { tube }\end{array}$ \\
\hline $\begin{array}{l}\text { Density }\left(\mathrm{kg} / \mathrm{m}^{3}\right) \\
\begin{array}{l}\text { Thermal Conductivity } \\
(\mathrm{W} / \mathrm{mK})\end{array}\end{array}$ & 2200 & 761 & 7850 \\
$\begin{array}{l}\text { Heat capacity at constant } \\
\text { pressure }(\mathrm{J} / \mathrm{kgK})\end{array}$ & 1000 & 0.121 & 44.5 \\
$\begin{array}{l}\text { Dynamic viscosity (Pas) } \\
\text { Pyna }\end{array}$ & - & 0.02 & 475 \\
\hline
\end{tabular}

Table 3. Boundary conditions used to solve the numerical model for the charging mode

\begin{tabular}{ll}
\hline Property & Value \\
\hline Initial temperature during charging, $T_{c, 0}$ & $350^{\circ} \mathrm{C}$ \\
Inlet temperature of the HTF during charging, $T_{c, \text { in }}$ & $390^{\circ} \mathrm{C}$ \\
Initial temperature during discharging, $T_{d, 0}$ & $390^{\circ} \mathrm{C}$ \\
Inlet temperature of the HTF during discharging, $T_{d, i n}$ & $350^{\circ} \mathrm{C}$ \\
Flow velocity of the HTF, $v$ & $0.25 \mathrm{~m} / \mathrm{s}$ \\
\hline
\end{tabular}

concrete is expressed as:

$$
\rho_{c} C_{p c} \frac{\partial T}{\partial t}=\nabla \cdot\left(k_{c} \nabla T\right)+Q^{\prime}
$$

where $C_{p, c}$ is the specific heat of concrete, $k_{c}$ is the thermal conductivity of concrete, $T$ is the temperature field in concrete and $Q^{\prime}$ is a source or sink term.

\subsection{Boundary conditions}

At $t=0 \mathrm{~s}, T=T_{c, 0}$ for the entire domain during the charging process, while $T=T_{d, 0}$ is set for discharging process. In order to simulate the charging and discharging, the flow direction of the heat transfer fluid is reversed so as the initial condition. CTES system is analyzed for only $3600 \mathrm{~s}$ for each case. The temperature of HTF at the inlet of the tube is set $T=T_{c, \text { in }}$ during charging, and during discharging $T=T_{d, i n}$. No slip boundary condition, $\mathbf{v}=0$ is applied to the tube wall. External insulation of the storage module is usually sufficiently thick to avoid the heat losses. All outer walls of the cylindrical concrete module are simulated as insulated walls by setting the boundary condition $\mathbf{n} \cdot k \nabla T=0$. Initial and boundary conditions used to solve the model for both sub-processes of the storage unit are given in Table 3 .

\subsection{Solution procedure}

The governing equations (Eqs.2-5) are valid for both charging and discharging process and are solved numerically with the appropriate initial and boundary conditions using a commercially available finite element method (FEM) software COMSOL v. 5.2a (Multiphysics, 2017). Sufficiently fine grid with 993785 and 1074263 elements is used to obtain the grid independent solution. Present model results are validated by the results achieved by Tamme et al. (2004). Figure 4 shows a 


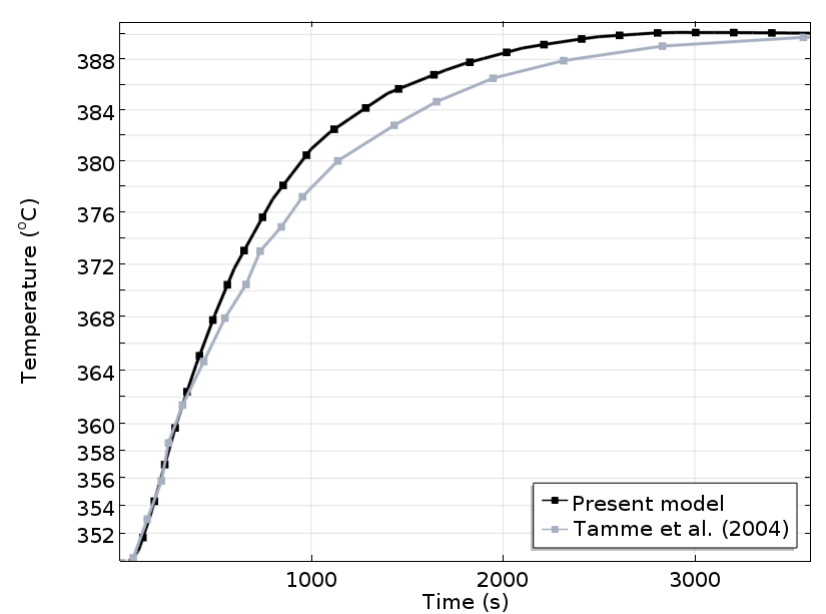

Figure 4. Comparison of storage temperature at distance $0.04 \mathrm{~m}$ from tube during charging

comparison between the average temperature at a distance $0.04 \mathrm{~m}$ in the storage predicted by the present model and that of Tamme et al. (2004). Reasonably good agreement between the results can be seen from the figure which confirms the reliability of the developed model.

\section{Results and discussion}

\subsection{Average temperature profile}

Figures $5 \mathrm{a}$ and $\mathrm{b}$ show a change in average temperature of the concrete storage unit with time during charging and discharging process. During charging, the thermal energy is stored as sensible heat which increases the average storage temperature with time. It can be seen that the system with finned tubes charges relatively faster than the system without finned tubes. At $t=3600 \mathrm{~s}$, the average temperature with bare tubes reaches $377^{\circ} \mathrm{C}$ while the temperature with finned tubes is observed $378^{\circ} \mathrm{C}$. Discharging of the storage systems can be seen in Fig.5b where the average temperature of the storage units decrease with time. Like charging process, the storage system with finned tubes tends to discharge relatively faster than the system with bare tubes. Note that in the present study, the system discharges from a fully charged state, i.e. from $T=390^{\circ} \mathrm{C}$. For better visualization, temperature distribution on a axial cross-sectional plane of the storage, with and without finned tubes, at different spans of time during the charging process is shown in Fig. 6. The dark blue contour lines around the tubes indicate the high temperature regions which spreads as the charging time progresses. The cooler region can be seen as green contour lines that exists close to the outer walls of the storage.

\subsection{Charging and discharging time}

During charging, heat is transferred from a higher temperature HTF to a lower temperature concrete storage while the reverse takes place in discharging. Implementation of fins on tubes increases the heat transfer rate and thus decreases charging and discharging period. Comparing time periods of the sub-processes provide a rational way to test the effectiveness of an enhancement method. It can be observed from Fig. 5a that finned tubes can considerably reduce the charging time. For instance, the storage system with finned tubes takes approximately $200 \mathrm{~s}$ less charging time to attain the average temperature of $366^{\circ} \mathrm{C}$ than the system without finned tubes. In other words, the charging time with finned tubes is reduced by approximately $50 \%$. The reduction, however, decreases with the charging time. For instance, to attain the average storage temperature of $377^{\circ} \mathrm{C}$, the charging time with finned tubes is reduced by approximately $11 \%$. Similar impact is observed in the case of discharging (Fig. 5b). It is worth to note that for a system to be cost-effective, the reduction in the charging time has to be balanced by additional cost appended by the fin material on the tubes that are used in the enhancement. The TES system is considered cost-effective if the cost of implementing finned tubes instead of bare tubes increase the production capacity for the application they are installed.

\subsection{Energy and exergy analysis}

Energy and exergy analysis is necessary to understand the thermodynamic behavior and the performance of thermal energy system. Energy based performance measures are often misleading, and do not reflect the useful energy correctly. Exergy analysis, on the other hand, helps to overcome such shortcomings of the energy analysis (Dincer and Rosen, 2002). In the present study, energy and exergy input during charging, and energy and exergy recovered during discharging are evaluated.

The amount of thermal energy stored or recovered in CTES system can be calculated using Eq.(1) as:

$$
E(t)=m C_{p}\left(T(t)-T_{0}\right)
$$

where $T(t)$ and and $T_{0}$ are the volume-averaged temperatures of the concrete module at any instance of time, $\mathrm{t}$ and at $t=0 \mathrm{~s}$, respectively. Note that losses from the system are considered negligible, and therefore, are not taken into account in the present study.

The exergy content at each time step of the simulation can be calculated by monitoring the volume-averaged temperature in the storage as (Dincer and Rosen, 2002):

$$
\xi(t)=m C_{p}\left[\left(T(t)-T_{0}\right)-T_{0} \ln \left(\frac{T(t)}{T_{0}}\right)\right]
$$

The impact of using finned tubes can be seen from the rate of charging and discharging the thermal energy in Figs.7a and $\mathrm{b}$. The change energy input during charging as a function of time can be seen in Fig. 7a. Within the charging time of $3600 \mathrm{~s}, 1.85 \mathrm{MJ}$ of thermal energy is stored by the system with bare tubes, whilst, the amount of energy stored by a system with finned tubes is $1.95 \mathrm{MJ}$. During the discharging, fully charged storage has $2.8 \mathrm{MJ}$ 


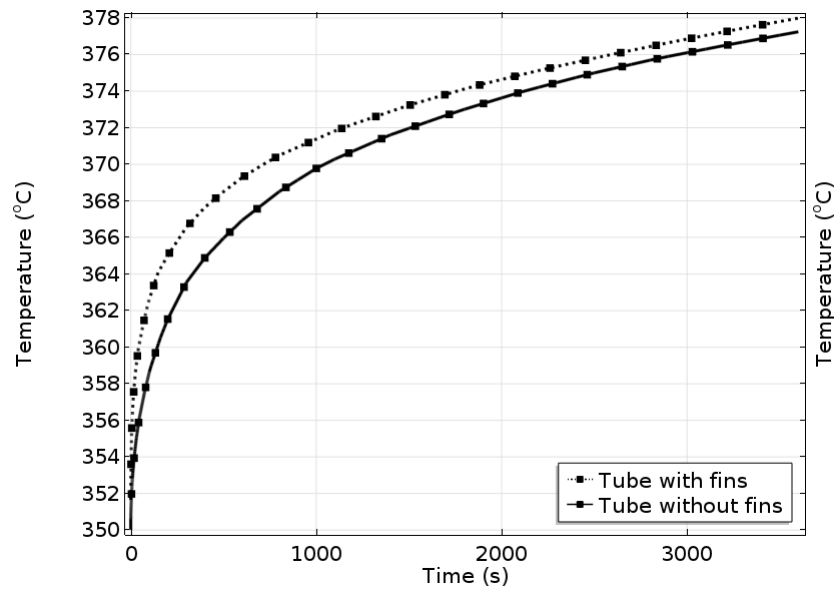

(a) Charging of CTES

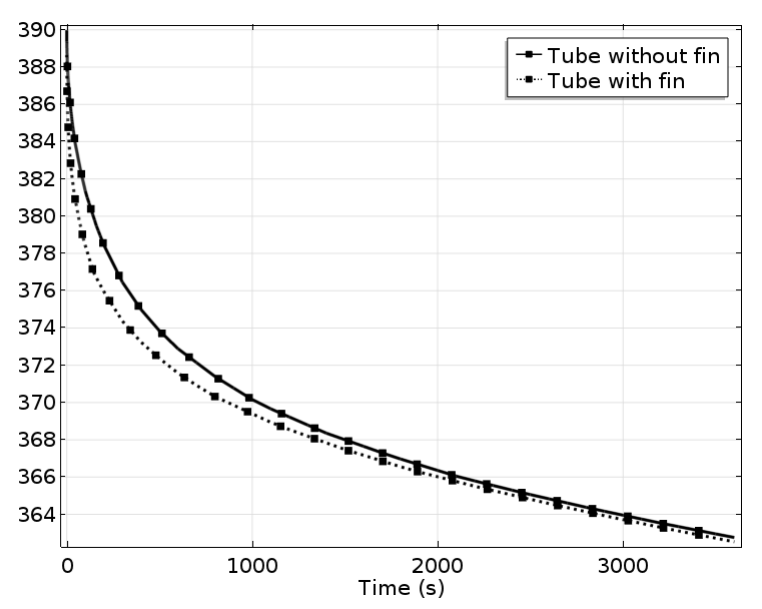

(b) Discharging of CTES

Figure 5. Variation of axial temperature of the concrete thermal energy storage system during sub-processes

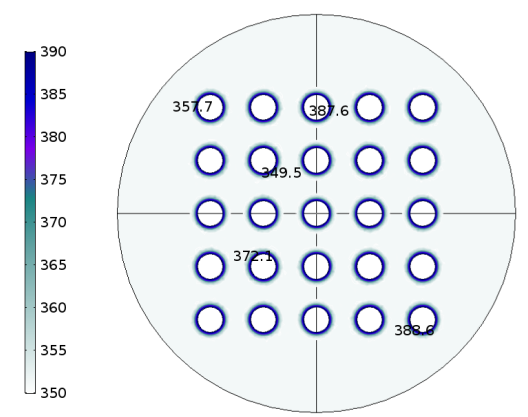

(a) Tube without fin, $\mathrm{t}=10 \mathrm{~s}$

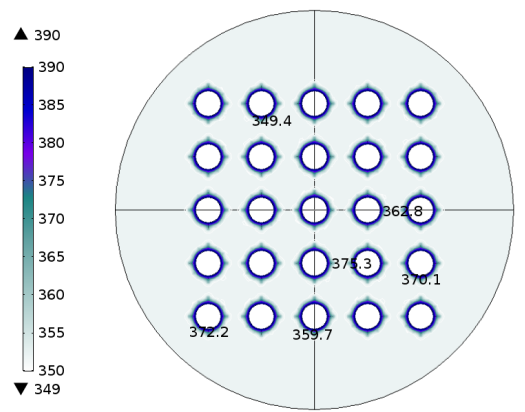

(d) Tube with fin,t=10s
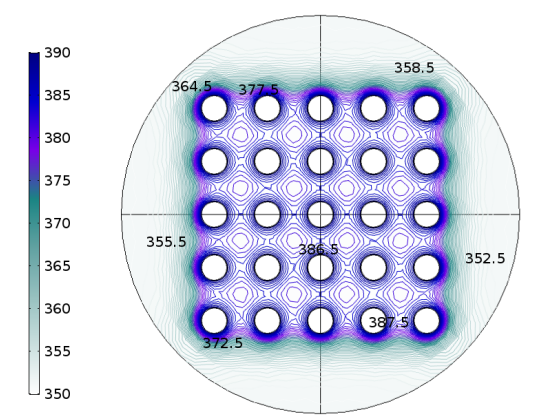

(b) Tube without fin, $\mathrm{t}=1000 \mathrm{~s}$
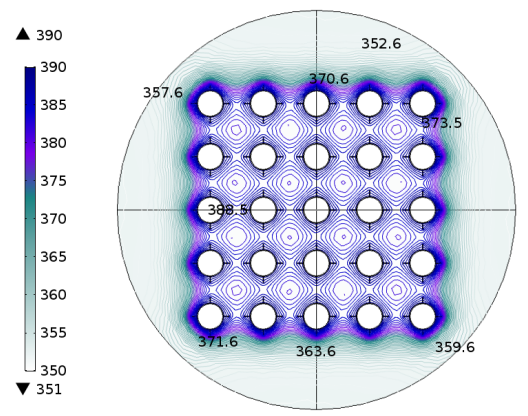

(e) Tube with fin,t=1000 s

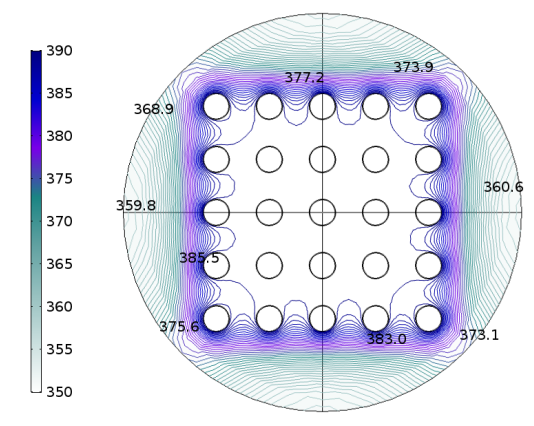

(c) Tube without fin, $\mathrm{t}=3600 \mathrm{~s}$

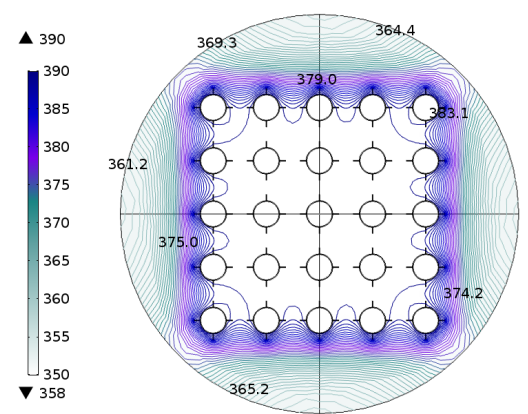

(f) Tube with fin,t=3600 s

Figure 6. Average temperature of the concrete thermal energy storage system with and without fins during charging

stored thermal energy which decreases with the discharging time as the HTF recovers it. Furthermore, the change in CTES exergy content during charging and discharging can be seen in Fig.8a and b, respectively.

\subsection{Energy and exergy efficiency}

Charging period energy efficiency can be defined as the ratio of energy accumulated in the CTES system to the input thermal energy during the charging process which can be mathematically expressed as (Dincer and Rosen,
2002):

$$
\eta_{c}=\frac{E(t)}{E_{0}}
$$

Exergy efficiency for charging period is defined as the ratio of exergy accumulation in CTES to the exergy input to CTES during the charging process and can be expressed as:

$$
\psi_{c}=\frac{\xi(t)}{\xi_{0}}
$$

Furthermore, energy and exergy efficiencies for discharging period is determined as the ratio of thermal 


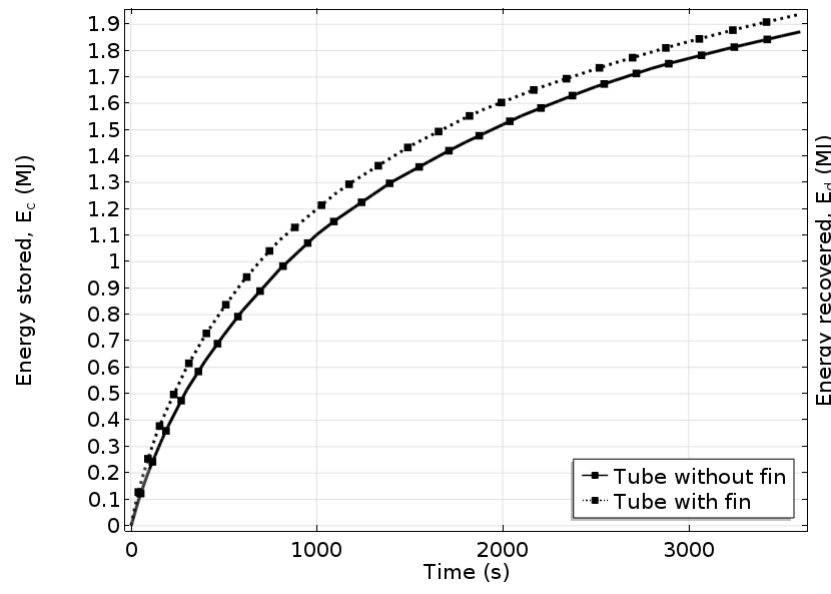

(a) Charging of CTES

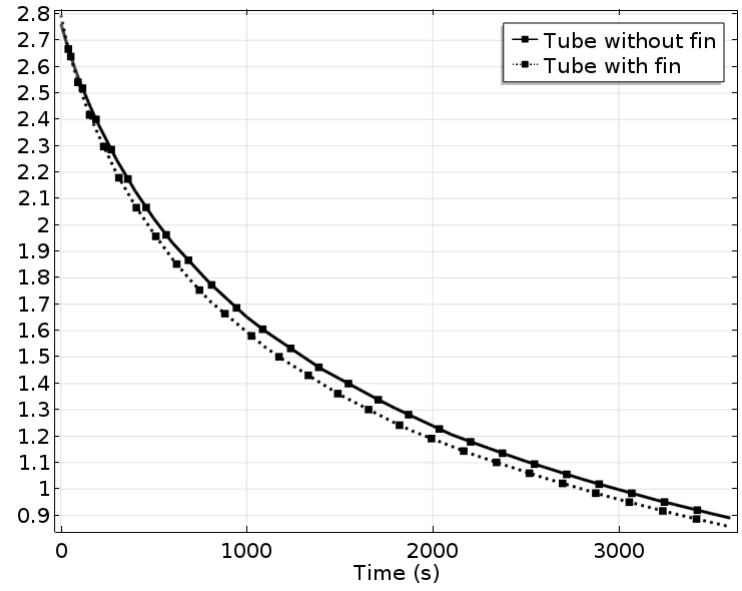

(b) Discharging of CTES

Figure 7. Change in thermal energy of concrete thermal energy storage system during sub-processes

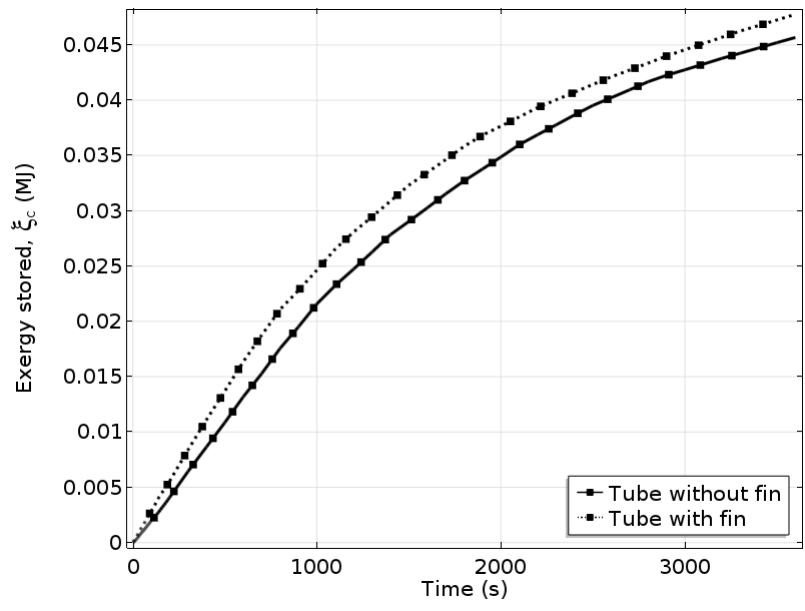

(a) Charging of CTES

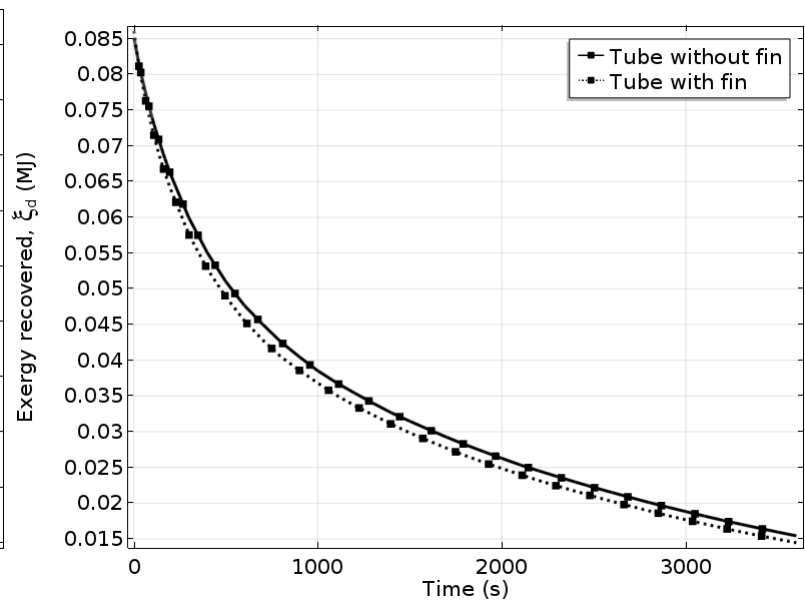

(b) Discharging of CTES

Figure 8. Change in exergy of the concrete thermal energy storage system during sub-processes

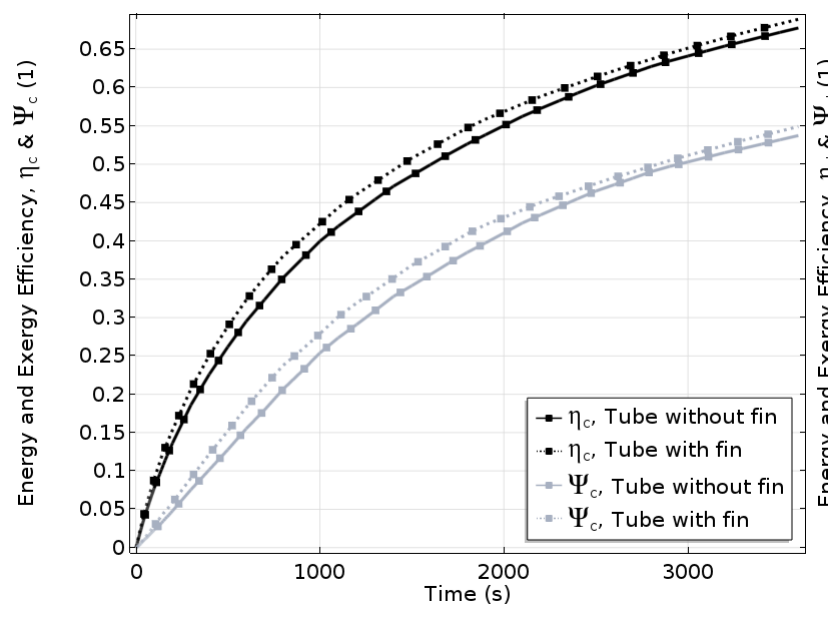

(a) Charging of CTES

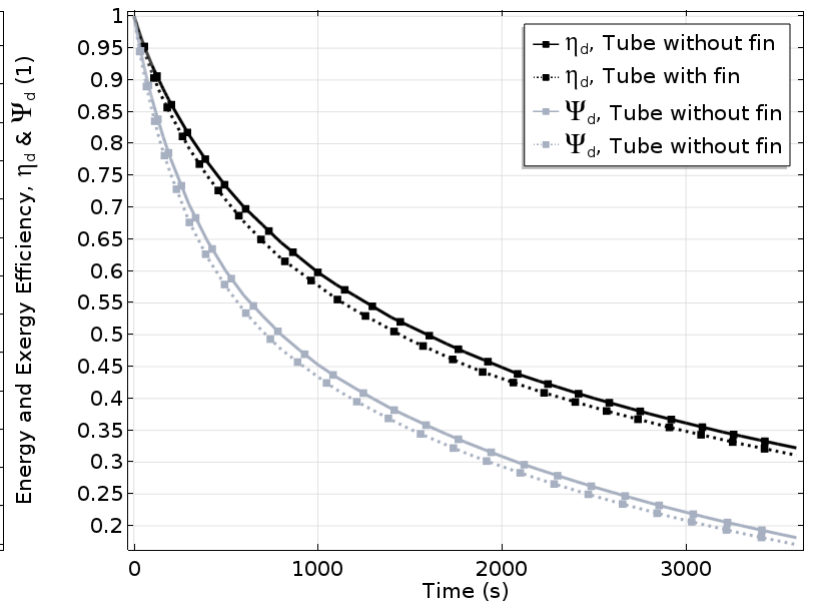

(b) Discharging of CTES

Figure 9. Energy and exergy efficiencies of the concrete thermal energy storage system during sub-processes 
energy or exergy recovered from CTES to the energy or exergy input to CTES. Efficiencies for both sub-processes are evaluated by monitoring the volume-averaged temperature within the storage system at each time step.

Figures $9 \mathrm{a}$ and $\mathrm{b}$ show the variation of energy and exergy efficiencies during charging and discharging of the CTES unit with and without finned tubes. The charging efficiency is zero at $t=0 \mathrm{~s}$ which increases with time due to the accumulation of thermal energy in the concrete. At charging time $t=3600 \mathrm{~s}$, the energy efficiency of the system without fins is observed $67.77 \%$. For the system with finned tubes, the charging energy efficiency is $68.90 \%$, which is slightly higher than the system without finned tubes. The efficiency can, however, be improved with optimized design and operating parameters. Due to negligible heat losses, the system would eventually attain charging efficiency of $100 \%$ where the storage is said to be fully charged. The exergy efficiency of the system is found lower than energy efficiency which shows a more meaningful value of the storage system performance that could be further improved by enhancement methods. It is apparent from Fig.9b that the thermal energy and exergy are being recovered from the fully charged mode during discharging period, therefore, both efficiencies decrease with time. From the results, it can be concluded that system with finned tubes has a relatively higher efficiency than the storage system without finned tubes.

\section{Conclusion}

The numerical investigation on concrete based thermal energy storage is carried out. Multiphysics model is developed to couple the heat transfer with the fluid flow in the storage system of heat exchanger tubes with and without fins. From the simulation results, the potential of finned tubes to enhance the heat transfer is determined in terms of storage temperature distribution, energy, and exergy efficiencies. For a given design of the storage system, selection of finned tubes can decrease the charging time by approximately $50-11 \%$. In addition, energy and exergy analysis concludes a considerable impact of finned heat exchanger tubes on energy and exergy efficiencies. The developed model can be employed as a framework to investigate the time-dependent performance of the CTES system integrated with a thermal power plant for steam generation. Efficient and cost-effective energy storage technology plays a key role in the grid integration of the renewables. Based on the present model, optimization of the design parameters such as number fins and tubes, heat exchanger tubes diameter that could significantly lower the investment cost for large scale storage capacity will be the focus of future studies.

\section{References}

F. Agyenim, P. Eames, and M. Smyth. A Comparison of Heat Transfer Enhancement in Medium Temperature Thermal
Energy Storage Heat Exchanger Using Fins and Multitubes. Solar Energy, 83(9):1509-1520, 2009. ISSN 0038092X. doi:10.1016/j.solener.2009.04.007.

I. Dincer and M. Rosen. Thermal energy storage: systems and applications. John Wiley \& Sons, 2002.

J. F. Feldhoff, K. Schmitz, M. Eck, L. Schnatbaum-Laumann, D. Laing, F. Ortiz-Vives, and J. Schulte-Fischedick. Comparative system analysis of direct steam generation and synthetic oil parabolic trough power plants with integrated thermal storage. Solar Energy, 86(1):520-530, 2012. ISSN 0038092X. doi:10.1016/j.solener.2011.10.026.

A. Gil, M. Medrano, I. Martorell, Ana Lázaro, P. Dolado, B. Zalba, and L. F. Cabeza. State of the art on high temperature thermal energy storage for power generation. Part 1-Concepts, materials and modellization. Renewable and Sustainable Energy Reviews, 14(1):31-55, 2010. ISSN 13640321. doi:10.1016/j.rser.2009.07.035.

D. Laing, W. D. Steinmann, R. Tamme, and C. Richter. Solid media thermal storage for parabolic trough power plants. Solar Energy, 80(10):1283-1289, 2006. ISSN 0038092X. doi:10.1016/j.solener.2006.06.003.

D. Laing, D. Lehmann, M. Fi, and C. Bahl. Test results of concrete thermal energy storage for parabolic trough power plants. Journal of Solar Energy Engineering, Transactions of the ASME, 131(4):410071-410076, 2009. ISSN 01996231. doi:10.1115/1.3197844.

D. Laing, C. Bahl, T. Bauer, and W. D. Lehmann, D.and Steinmann. Thermal energy storage for direct steam generation. Solar Energy, 85(4):627-633, 2011. ISSN 0038092X. doi:10.1016/j.solener.2010.08.015.

M. Medrano, A. Gil, I. Martorell, X. Potau, and L. F. Cabeza. State of the art on high-temperature thermal energy storage for power generation. Part 2-Case studies. Renewable and Sustainable Energy Reviews, 14(1):56-72, 2010. ISSN 13640321. doi:10.1016/j.rser.2009.07.036.

COMSOL Multiphysics. COMSOL Multiphysics $®$. Technical report, https://www.comsol.com/, 2017.

M. Seitz, P. Cetin, and M. Eck. Thermal storage concept for solar thermal power plants with direct steam generation. Energy Procedia, 49:993 - 1002, 2014. ISSN 1876-6102. doi:http://dx.doi.org/10.1016/j.egypro.2014.03.107.

R. Tamme, D. Laing, and W. D. Steinmann. Advanced Thermal Energy Storage Technology for Parabolic Trough. 126(2): 794-800, 2004. doi:10.1115/1.1687404. 